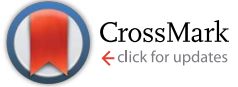

Cite this: RSC Adv., 2017, 7, 11913

Received 2nd November 2016 Accepted 10th February 2017

DOI: 10.1039/c6ra26195g

rsc.li/rsc-advances

\section{Enhancement of the carrier mobility of conducting polymers by formation of their graphene composites}

\begin{abstract}
A. K. Chauhan, ${ }^{\star a}$ S. K. Gupta, ${ }^{a}$ D. Taguchi, ${ }^{\text {b }}$ T. Manaka, ${ }^{\text {b P. Jha, }}{ }^{\text {a P. Veerender, }}{ }^{a}$
C. Sridevi, ${ }^{a}$ S. P. Koiry, ${ }^{a}$ S. C. Gadkari ${ }^{a}$ and M. Iwamoto ${ }^{b}$

Conducting polymers (CP) with high charge carrier mobility are crucial for flexible organic electronic devices. However, the inherent carrier mobility of these polymers is very low. Therefore, methodologies need to be explored to improve the carrier transport in these polymers so that they can be efficiently used in organic electronic devices. Graphene, due to its exceptional electrical and mechanical properties, is a promising material to be examined for its possible incorporation in CP matrix to achieve the objective. We have prepared graphene composites of the conducting polymers following an optimized procedure and these were investigated for their charge transport properties. The mobility values were measured using electric field induced second harmonic generation (EFISHG) and field effect transistor (FET) transfer characteristics. Both the transient and average mobilities were found to increase significantly with the inclusion of graphene. This enhancement in mobility has been attributed to an ordered packing of the thinner and smaller graphene sheets with polymer chain and interfacial $\pi-\pi$ interaction. To substantiate its usefulness in device applications, the effect of graphene inclusion was also investigated for polymer solar cells and it was observed that despite of reduction in open circuit voltage, device fabricated using graphene composites yielded about $20 \%$ higher efficiencies as compared to pristine conducting polymer devices.
\end{abstract}

\section{Introduction}

Conducting polymers have several advantages over conventional semiconductors like mechanical flexibility, easy processing and transparency which can help in fabrication of cheaper flexible electronic devices. ${ }^{\mathbf{1}}$ The research on conducting polymers has been recently intensified for fabrication of various cheap and lightweight organic devices, such as LEDs, ${ }^{2}$ solar cells, ${ }^{3}$ transistors, energy storage, ${ }^{4}$ thermoelectric, ${ }^{5}$ rechargeable batteries, ${ }^{6}$ and chemical/biosensors. ${ }^{7}$ In most of these polymers, free charge carriers are created on absorption of light and hence they are widely used in bulk heterojunction solar cells. ${ }^{\mathbf{8} 9}$

Poly(3-hexylthiophene) (P3HT) and poly[ $N$-9'-heptadecanyl2,7-carbazole-alt-5,5-(4',7'-di-2-thienyl-2', $1^{\prime}, 3^{\prime}$-benzothiadiazole)] (PCDTBT) have been among the most sought conducting polymer materials for organic photovoltaic applications owing to their strong optical absorption and easy processibility from solution. ${ }^{\mathbf{1 0 , 1 1}}$ However, their typical charge carrier mobility is very low and need to be improved for efficient devices. The

${ }^{a}$ Technical Physics Division, Bhabha Atomic Research Centre, Trombay, Mumbai, 400085 India. E-mail: akchau@barc.gov.in; akc.barc@gmail.com; Tel: +91-22-25593911

${ }^{b}$ Department of Physical Electronics, Tokyo Institute of Technology, O-okayama, Meguro-ku, Tokyo, 152-8552, Japan energy bands in conducting polymers are extremely narrow and charge transport is facilitated via hopping, as a result the motion of carriers slows down resulting in low mobility that in turn severely hampers their practical application. To improve the charge transport, research has been continuously developing and an increasing interest was devoted to hybrid assemblies based on CPs. Attempts are reported where CPs in combination with a variety of materials like non-conducting polymers, metals, metal oxides/chalcogenides, CNTs, carbonaceous materials, and other inorganic compounds were tried and investigated by several groups. ${ }^{\mathbf{1 2 - 1 6}}$ However, the success of these attempts have remain limited.

Graphene is a semimetal where conduction and valence band coincide at the Fermi level. It exhibits a strong ambipolar electric field effect and resembles a semiconductor having a charge carrier concentration up to $10^{13} / \mathrm{cm}^{2}$. Graphene sheets consist of $\mathrm{sp}^{2}$-bonded carbon atoms arranged in one-atom thick hexagonal lattices and exhibits unique characteristics of extraordinary electron mobility, intrinsic material strength, and thermal properties. In view of the above, graphene based composites are emerging as a promising class of materials for several applications and when functionalized graphene (or graphene oxide) incorporated into polymers some of these properties manifest a remarkable improvement in the host material. ${ }^{17-25}$ However, incorporation of pristine graphene 
sheets into conducting polymer matrix is quite complex, as during the formation process, typically a few layer thick and few micron long graphene flakes are produced and these can provide a direct path for carrier transport to overturn the purpose. Therefore, an approach for making the composites using smaller sheets is required.

We have successfully prepared graphene composites of these polymers using thin and short graphene sheets and investigated their carrier transport properties. In this paper, we demonstrate a significant improvement in mobilities of these composites using direct visualization of carrier movement and also analyze the reasons for this improvement. Solar cells were fabricated using these composites and their performances were also investigated and reported.

\section{Experimental}

PCDTBT, P3HT, and phenyl- $\mathrm{C}_{61}$-butyric acid methyl ester (PCBM) were obtained from $\mathrm{M} / \mathrm{s}$ Aldrich Chemicals and used without further purification. Solution of short graphene-sheets was obtained by keeping the suspension of commercially available graphene-flakes in dichlorobenzene $(0.1 \mathrm{mg} / 10 \mathrm{~mL})$ for 30 days in an ultrasonic bath. The ultrasonic agitation resulted in the exfoliation of thin and short graphene sheets. ${ }^{26}$ The structural characterization of obtained graphene sheets was carried out using Raman spectroscopy (model LabRam HR100) and transmission electron microscopy (model Carl Zeiss Libra(R) 200FE).

For the purpose of transport studies, the active layer solutions were prepared by dissolving P3HT and PCDTBT $(20 \mathrm{mg}$ $\mathrm{mL}^{-1}$ and $10 \mathrm{mg} \mathrm{mL}^{-1}$ respectively) in dichlorobenzene. The solutions were stirred in dark for $48 \mathrm{~h}$ and then filtered using a 0.45 micron filter. In order to make composites, solution of exfoliated graphene sheets was blended with the conducting polymer solution $\left(10 \mu \mathrm{L} \mathrm{mL}{ }^{-1}\right)$ for $48 \mathrm{~h}$ and a homogeneous blend was obtained. Homogeneity of the blend was confirmed using Raman spectroscopy on three sets of deposited films at different places.

The carrier mobilities were measured using the electric field induced second harmonic generation (EFISHG) and organic field effect transistor (OFET) transfer characteristics. For these studies, the sample structures were fabricated in bottom gate top contact FET geometry on commercially available Si substrates having thermally grown $500 \mathrm{~nm} \mathrm{SiO}_{2}$ layer. The basic arrangement of the experimental system is shown in Fig. 1, where a laser beam (from femtosecond optical parametric amplifier pumped by a Ti:sapphire regenerative amplifier system) was vertically incident onto the sample through the objective lens and images formed by SHG light from the samples were captured at $550 \mathrm{~nm}$ for different time intervals using a CCD camera.

The transfer ( $I_{\mathrm{DS}} v s$. $V_{\mathrm{GS}}$ at constant $\left.V_{\mathrm{DS}}\right)$ characteristics of the fabricated transistors were measured using a setup containing two source-meters and in-house developed software. Field effect hole mobility values (in saturation region) were calculated from the OFET transfer characteristics following an established procedure. $^{27,28}$
In order to study the photovoltaic characteristics, the solar cells were fabricated using P3HT/PCDTBT as donor polymer and PCBM as an acceptor molecule. The active layer blend was prepared in dichlorobenzene solvent by mixing of $\mathrm{P} 3 \mathrm{HT} /$ PCDTBT and PCBM in $1: 1$ and $1: 2$ weight ratio respectively. For fabrication of solar cell devices ITO coated glass having 10 Ohm per square resistivity was taken and patterned into three linear strips size $3 \mathrm{~mm} \times 25 \mathrm{~mm}$ separated by $\sim 4 \mathrm{~mm}$ using chemical treatment by etching out the undesired area using an optimized acidic solution. Such prepared substrates were cleaned using doubly distilled deionized water (resistivity $\sim 18.2 \mathrm{M} \Omega \mathrm{cm}$ ), acetone, iso-propanol and dried by blowing pressurized nitrogen, followed by UV-ozone treatment for 15 minutes prior to deposition of Hole Transport Layer (HTL). $50 \mathrm{~nm}$ layer of poly(3,4-ethylenedioxythiophene) poly (styrenesulfonate) (PEDOT:PSS) (Ossila-PH-1000) was spin casted on the substrate at $2500 \mathrm{rpm}$ and annealed for $2 \mathrm{~h}$ at $120^{\circ} \mathrm{C}$. Subsequently, the PEDOT:PSS deposited substrates were transferred inside a glove box (MB 20G, MBraun Inc. Germany) where oxygen and moisture were controlled within $0.1 \mathrm{ppm}$ level. The active layer blend was spin casted at a spinning speed of $2000 \mathrm{rpm}$ in the glove box. Finally, $100 \mathrm{~nm}$ aluminum electrodes were deposited by thermal evaporation (vacuum: $1 \times$ $10^{-6}$ Torr) using a shadow mask to yield $3 \times 3$ array of devices each cell bearing an area of $6 \mathrm{~mm}^{2}$. The typical device structure is shown in Fig. 1c. Prior to transferring the device outside glove box, it was sealed using an epoxy (Ossila) and was cured by UV treatment. The photovoltaic measurements were carried out on AAA Solar Simulator (Sciencetech SS 150) calibrated using NREL certified standard Si solar cell.

\section{Results \& discussion}

For structural characterization, TEM images and Raman spectrum of exfoliated graphene sheets were recorded and presented in Fig. 2. The TEM images revealed that typical size of nanosheets is $\sim 25 \mathrm{~nm}$. The Raman spectra exhibit two principal bands ( $G$ and 2D) for graphene and a third $D$ band related to defects. The symmetry of $2 \mathrm{D}$ peak indicates presence of pure graphene. The intensity ratio of $2 \mathrm{D}$ and $\mathrm{G}$ band is found to be $\sim 0.5$ which shows that exfoliated sheets typically contain 3-5 layers. ${ }^{29-31}$ The D band peak requires defects for its activation and its presence and reasonable intensity indicates the existence of defects from the edges and thus reconfirms presence of short sheets.

The movement of injected carriers can be directly visualized using EFISHG measurements. ${ }^{32-34}$ The recorded SHG images at different time intervals are presented in Fig. 3. On application of a step voltage pulse (of $250 \mu$ s width), carriers are injected from the source electrode into the organic semiconductor layer and transported between the two electrodes. Due to the presence of these carriers an electric field is generated inside the organic semiconductor resulting in the emission of SHG light due to nonlinear susceptibility of the organic compounds. The actual locations of carrier (holes) at a certain time after the application of a $70 \mathrm{~V}$ pulse are presented in Fig. 3 in form of snapshots. Set a, b, c, and d, are for P3HT, P3HT:graphene, 


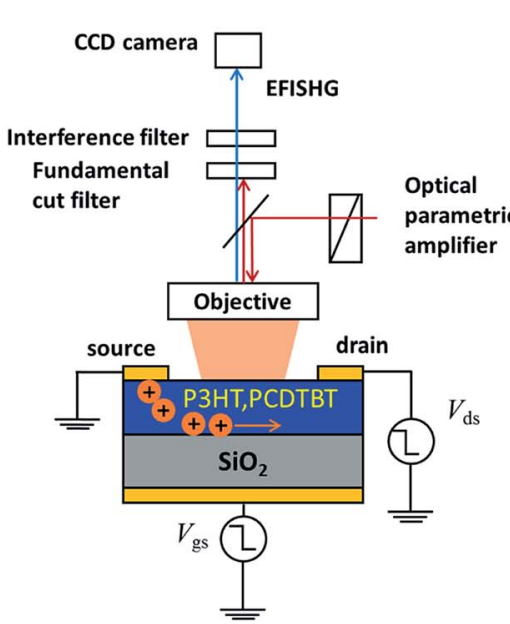

(a)

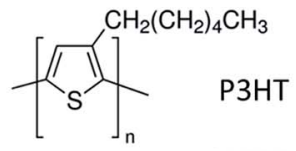

PCDTBT

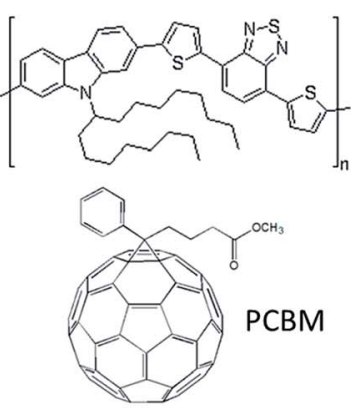

(b)

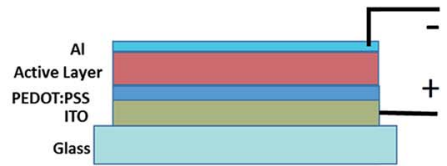

(c)

Fig. 1 (a) Setup for EFISHG measurement (b) chemical structure of P3HT, PCDTBT, PCBM and (c) typical solar cell structure.

PCDTBT, and PCDTBT:graphene respectively. The location and intensity of the bright cloud indicates the presence and concentration of the holes at any point of time. A bright horizontal line towards the bottom is the position of the source electrode. As the time passes, this cloud progressively moves upward towards the drain electrode. The darker spots/areas in these images could be attributed to morphology of the deposited films as emitted light did not have an oblique emission and hence the light was not collected by the detector. It is evident from the figures that the injection of carriers is very smooth in all the films. A close observation of these pictures reveals that initially the carrier movement is quick however, as they move away from the electrodes their motion becomes relatively slow. This could be attributed to the reduction in coulombic repulsion owing to the change in concentration of the carriers over the period of time, in addition to presence of structural disorder, defects, trapping centers, and poor interconnectivity. The mobility values at different times calculated from the actual movement of their wave-front are presented in Table 1.
For pure P3HT samples (Fig. 3a), the carrier movement is slow as compared to graphene composite (Fig. 3b). In P3HT sample carriers are seen approaching the drain electrode in $\sim 10$ $\mu$ s time. In both the cases, as the movement progresses the sharpness is faded and towards the end the cloud is uniformly distributed. It suggests an even distribution of the carriers and also indicate that some holes are still exist near the source electrodes. The mobility value in graphene composite as compared to pristine P3HT is increased by an order from $4.0 \times$ $10^{-3}$ to $4.2 \times 10^{-2} \mathrm{~cm}^{2} \mathrm{~V}^{-1} \mathrm{~s}^{-1}$ (as calculated after $0.2 \mu \mathrm{s}$ of injection). This method provides the precise position of carriers in real space and time and thus measures the transient mobility. It is evident that the value changes with distance travelled after injection. For the comparison of values a moderate time of $0.2 \mu \mathrm{s}$ was chosen.

The transient mobility value of pure PCDTBT films was measured $3.6 \times 10^{-3} \mathrm{~cm}^{2} \mathrm{~V}^{-1} \mathrm{~s}^{-1}$ which was marginally less than pure P3HT films. On addition of graphene in the film the mobility was enhanced to $6.1 \times 10^{-3} \mathrm{~cm}^{2} \mathrm{~V}^{-1} \mathrm{~s}^{-1}$. For the
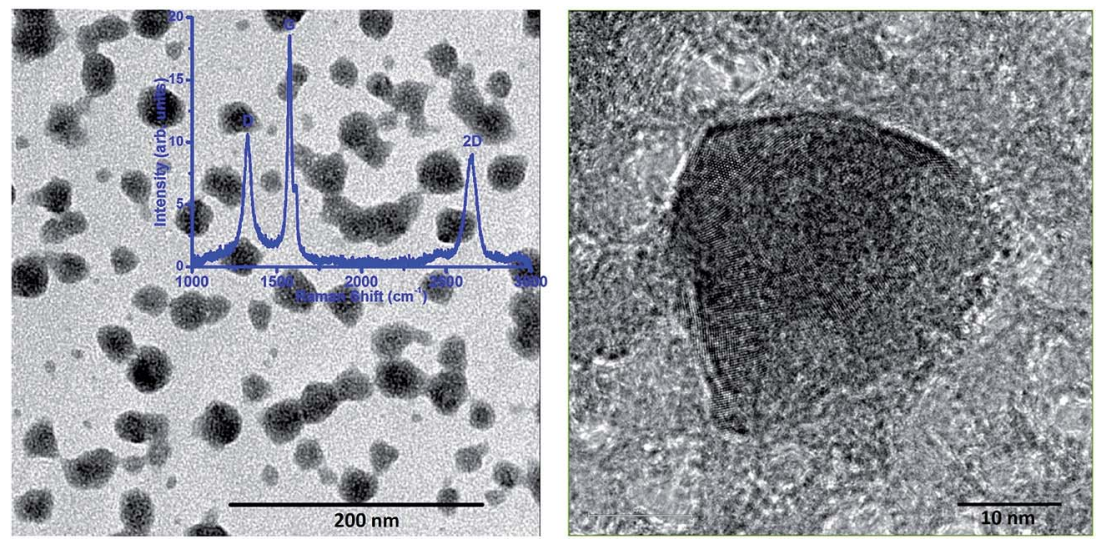

Fig. 2 (a)TEM Images of the exfoliated graphene nano-sheets, inset shows recorded Raman spectrum where the characteristic graphene D, G and $2 \mathrm{D}$ bands are visible at 1340, 1580 and $2670 \mathrm{~cm}^{-1}$ respectively (b) high resolution image of a single nano sheet indicating the typical sheet size of $\sim 25 \mathrm{~nm}$. 
(a)
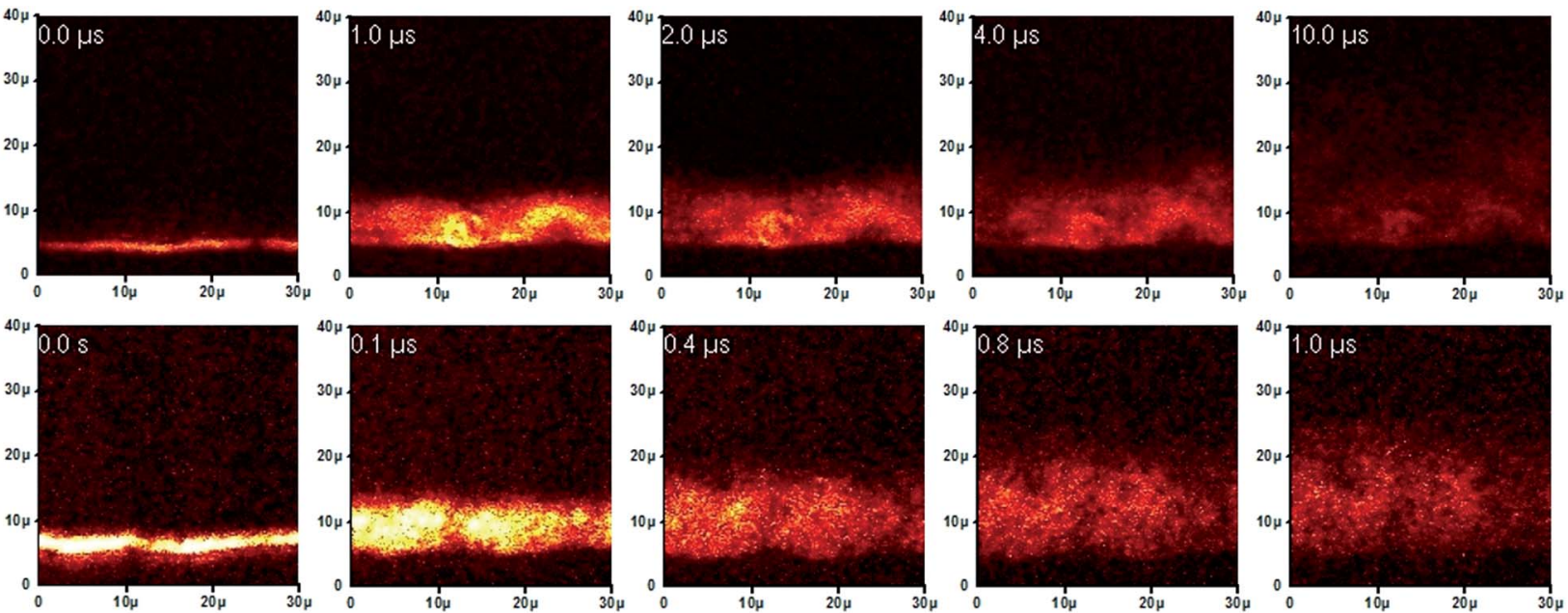

(b)
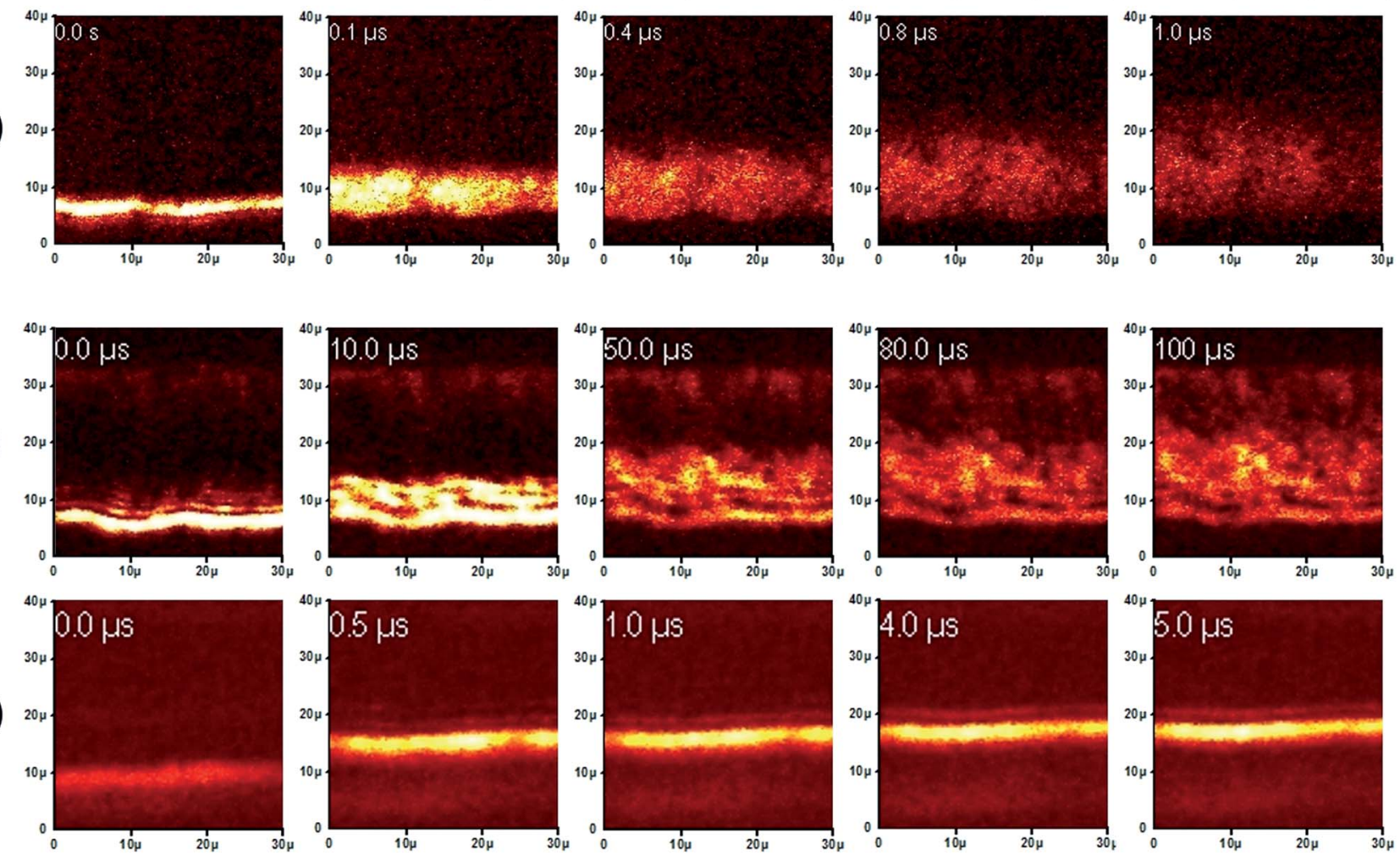

Fig. 3 Visualization of hole movements through emission of $550 \mathrm{~nm}$ wavelength for (a) P3HT, (b) P3HT:graphene, (c) PCDTBT, and (d) PCDTBT:graphene films at the indicated time after application of $70 \mathrm{~V}$ pulse to the electrodes with $30 \mu \mathrm{m}$ channel width.

reasons described earlier, the transient mobilities were found to reduce with the time and after $2 \mu$ s of the injection, the mobility value was reduced to $1.4 \times 10^{-3} \mathrm{~cm}^{2} \mathrm{~V}^{-1} \mathrm{~s}^{-1}$ for pristine and 1.8 $\times 10^{-3} \mathrm{~cm}^{2} \mathrm{~V}^{-1} \mathrm{~s}^{-1}$ for graphene composite, which is similar behavior as observed for the P3HT films. The reason for a sharp drop in mobility value is that the carriers have to hop from one crystalline domain to another in order to get transported over entire distance. In addition, structural disorder, defects, and poor interconnectivity also play their role to trap charges and reduced mobility during this movement in conducting polymers..$^{35}$ To further investigate the charge transport, average mobility values were also measured by FET transfer characteristics. The calculated mobility values are given in Table 1 . The averaged mobility for graphene composite with P3HT has been enhanced about three fold from $1.6 \times 10^{-4}$ to $5.7 \times 10^{-3} \mathrm{~cm}^{2} \mathrm{~V}^{-1} \mathrm{~s}^{-1}$. However, in case of PCDTBT the mobility was increased from $4.7 \times 10^{-5}$ to $7.9 \times 10^{-5} \mathrm{~cm}^{2} \mathrm{~V}^{-1} \mathrm{~s}^{-1}$. As expected, these are found lower than the transient mobilities.

There could be following three possible reasons for the enhancement of mobility in the composite films: (a) either graphene itself is contributing to the conductivity, (b) a chargetransfer doping of the organic semiconductor is taking place and (c) there is an improvement in the interior morphology of the films due to incorporation of graphene, which eventually is

Table 1 Measured mobilites in $\mathrm{cm}^{2} \mathrm{~V}^{-1} \mathrm{~s}^{-1}$ using EFISHG methods and OFET transfer curves

\begin{tabular}{|c|c|c|c|c|c|}
\hline Method & \multicolumn{2}{|l|}{ Р3НT } & \multicolumn{3}{|l|}{ PCDTBT } \\
\hline With graphene & $3.7 \times 10^{-2}$ & $1.6 \times 10^{-4}$ & $6.1 \times 10^{-3}$ & $1.8 \times 10^{-3}$ & $7.9 \times 10^{-5}$ \\
\hline
\end{tabular}


reflected in form of mobility enhancement. The AFM images (not presented here) have shown that inclusion of graphene has not made any noticeable contribution to the average roughness which remain of the order of few nanometers and therefore third possibility (c) is ruled out, and others are discussed below.

Both P3HT and PCDTBT have an inherently unstable backbone of a finite length resulting from the formation of alternate single and double bonds along the chains and the mobile carriers are added through doping. The delocalized pi-bonding electrons across the conjugated back bone provide a pathway for the movement of these carriers. It is prudent to mention here that the electronic energy bands are extremely narrow and slow down the carrier motion substantially. These slow moving electrons distort/polarize the lattice and polarons are formed with long life time. ${ }^{36}$ The added layer of graphene is a zerooverlap semimetal and contains both holes and electrons as charge carriers. After two dimensional bonding through $\mathrm{sp}^{2}$ hybridization one electron is left freely available in the third dimension which forms a set of protruding $\pi$ electrons clouds in out of the plane direction. These highly-mobile electrons are located above and below the graphene sheets and the electronic properties of graphene are dictated by the bonding and antibonding of these $\pi$ orbitals. When a thinner graphene sheet comes into close contact with P3HT and PCDTBT, their conducive surface energies organize them to get arranged in a lamellar packing and would facilitate a $\pi-\pi$ interaction between the chains. ${ }^{37,38}$ Under this situation, the interaction of their $\pi$-orbitals in singlet state will distort the crystal potential and create additional localized levels near to highest occupied molecular orbital (HOMO) and lowest unoccupied molecular orbital (LUMO) in the forbidden region. This enforce creation of an additional polaron in the conducting polymer. ${ }^{39}$ Thus, the interaction of graphene with $\mathrm{P} 3 \mathrm{HT}$ and PCDTBT creates additional polarons in the lattice through a $\pi-\pi$ interaction and tune the electrical properties. ${ }^{40}$ The similar improvement in the mobility of conjugated polymers has been reported and attributed to the formation of an ordered lamellar packing of the alkyl side chains benefitting the inter-chain $\pi-\pi$ interaction, ${ }^{41}$ which is graphene in the present case. The typical size of graphene sheets is very small as compared to long polymer chain, the role of additional electrons available with graphene over the entire pathway is limited and therefore, the first possibility (a) is also less probable. Therefore, it is proposed that in the presence of graphene, availability of the additional holes across the chain for conduction enhance the mobility of conducting polymer chain. It may be noted that researchers have demonstrated the similar improvement in mobility by adding $\sim 10 \%$ of functionalized CNTs and addition to such large amount creates structural deformation. However, in our case, PPM level of graphene has made a significant enhancement. ${ }^{42,43}$

The measured $J-V$ characteristics of different devices are shown in Fig. 4 and calculated photovoltaic parameters are presented in Table 2. When graphene composite was used in place of PCDTBT, the open circuit voltage $\left(V_{\text {oc }}\right)$ was reduced from $0.66 \mathrm{~V}$ to $0.63 \mathrm{~V}$, however the short circuit current $\left(J_{\mathrm{sc}}\right)$ was increased from $14 \mathrm{~mA} \mathrm{~cm}^{-2}$ to $17 \mathrm{~mA} \mathrm{~cm}^{-2}$ and fill factor (FF) was increased marginally. A similar trend was observed for P3HT:PCBM solar cells where $V_{\text {oc }}$ was reduced from 0.63 to $0.59 \mathrm{~V}$ and $J_{\mathrm{sc}}$ was increased from 12 to $13 \mathrm{~mA} \mathrm{~cm}^{-2}$. As a result, when graphene composites were used an overall improvement of $\sim 20 \%$ for PCDTBT and $\sim 10 \%$ for P3HT based devices have been observed. When similar experiments were performed with larger graphene flakes the device quality was degraded. It is reported that blending of graphene with $\mathrm{P} 3 \mathrm{HT}$ has yielded an efficiency to
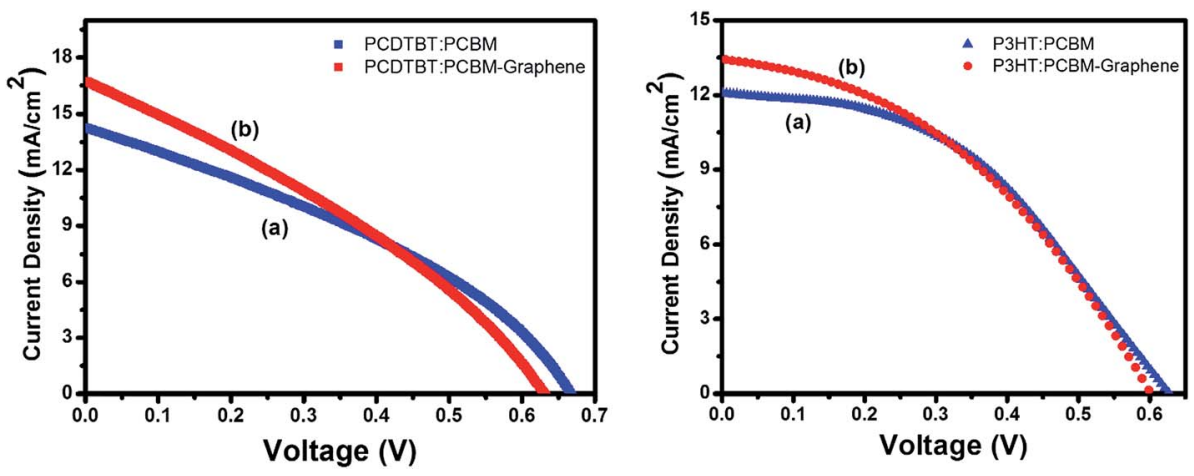

Fig. $4 J-V$ characteristics of PCDTBT and P3HT based solar cell devices under 1 Sun illumination.

Table 2 Extracted photovoltaic parameters for PCDTBT and P3HT solar cells

\begin{tabular}{lllll}
\hline & \multicolumn{2}{c}{ PCDTBT:PCBM } & & \multicolumn{2}{c}{ P3HT:PCBM } \\
\cline { 2 - 3 } & Without graphene & With graphene & Without graphene & $0.63 \pm 0.01$ \\
\hline$V_{\text {oc }}($ Volts $)$ & $0.66 \pm 0.01$ & $0.63 \pm 0.01$ & 12 & $0.59 \pm 0.01$ \\
$I_{\text {sc }}\left(\mathrm{mA} \mathrm{cm}^{-2}\right)$ & 14 & 17 & 42 & 13 \\
Fill factor $(\%)$ & 28 & 30 & 3.2 & 3.2
\end{tabular}


$1.1 \%$ (ref. 44 ) our results shows that thinner and shorter sheets are more beneficial owing to their lamellar packing.

In general, $V_{\mathrm{oc}}$ originates due to the splitting of electron and hole quasi-Fermi energy levels actuated by light illumination. ${ }^{45}$ However, under practical conditions, the charge transfer states originated at the $\mathrm{D}-\mathrm{A}$ interface also plays a major role. ${ }^{\mathbf{4 6 , 4 7}}$ Charge Transfer (CT) state complexes (also known as polaron pairs) are interfacial electron-hole pairs located at the donoracceptor (D/A) hetero-interface ${ }^{48-52}$ and are closely related to polaron pairs. ${ }^{52}$ Also, there exist a direct relationship between $V_{\text {oc }}$ and average energy of CT states. ${ }^{53}$ The CT states will affect the $V_{\mathrm{oc}}$ when the exciton would populate these states provided the levels are lower to the singlet state. The interaction of graphene with donor polymers could downshift the electron quasiFermi level and upshift the hole quasi-Fermi level thereby reducing the $V_{\text {oc }}$ correspondingly.

If the recombination rate remains unaltered, enhanced mobility in the presence of graphene could facilitate the faster collection of holes at anode, which eventually improves $J_{\mathrm{sc}}$. Thus the improvement in $J_{\mathrm{sc}}$ has been attributed to better mobility values. In all, the current has increased owing to better charge transport, however, a reduction in open circuit voltage has offset some of its effect on efficiencies. Nevertheless there is a definite improvement ( 10-20\%) in the device efficiencies.

\section{Summary}

To summarize, graphene has been used to improve the carrier mobility in the polymers namely P3HT and PCDTBT. The graphene composites were prepared under optimized condition after exfoliation of thinner and shorter graphene sheets from nano-flakes. The charge transports in these composites were mapped using EFISHG and further investigated by measuring the mobility values using FET transfer characteristics. The carrier injection was smooth and both the average and transient mobility values were found to have significantly increased in graphene composites. The enhancement in mobility has been attributed to an ordered lamellar packing of the graphene with polymer chain and interfacial $\pi-\pi$ interaction. The graphene composites solar cells were found to yield better efficiencies owing to improved carrier transport. To conclude, we have demonstrated that shorter and thinner graphene sheets are suitable for making their composites with conducting polymers which bears remarkably improved mobility values.

\section{Acknowledgements}

This work is supported by DST-JSPS Indo-Japan collaborative research project (DST/INT/JSPS/P-198/2015) granted to Prof. S. K. Gupta and Prof. M. Iwamoto.

\section{References}

1 S. R. Forrest, The path to ubiquitous and low-cost organic electronic appliances on plastic, Nature, 2004, 428(6986), 911-918.
2 J. H. Burroughes, D. D. C. Bradley, A. R. Brown, R. N. Marks, K. Mackay, R. H. Friend, et al., Light-Emitting-Diodes Based On Conjugated Polymers, Nature, 1990, 347(6293), 539-541.

3 M. Skompska, Hybrid conjugated polymer/semiconductor photovoltaic cells, Synth. Met., 2010, 160(1), 1-15.

4 Y. G. Li, W. Zhou, H. L. Wang, L. M. Xie, Y. Y. Liang, F. Wei, et al., An oxygen reduction electrocatalyst based on carbon nanotube-graphene complexes, Nat. Nanotechnol., 2012, 7(6), 394-400.

5 Z. U. Khan, J. Edberg, M. M. Hamedi, R. Gabrielsson, H. Granberg, L. Wågberg, et al., Thermoelectric Polymers and their Elastic Aerogels, Adv. Mater., 2016, 28, 4556-4562.

6 G. Wang, L. Zhang and J. Zhang, A review of electrode materials for electrochemical supercapacitors, Chem. Soc. Rev., 2012, 41(2), 797-828.

7 D. W. Hatchett and M. Josowicz, Composites of intrinsically conducting polymers as sensing nanomaterials, Chem. Rev., 2008, 108(2), 746-769.

8 A. Facchetti, $\pi$-conjugated polymers for organic electronics and photovoltaic cell applications, Chem. Mater., 2010, 23(3), 733-758.

9 A. J. Heeger, Semiconducting polymers: the third generation, Chem. Soc. Rev., 2010, 39(7), 2354-2371.

10 Y. Zhang, E. Bovill, J. Kingsley, A. R. Buckley, H. Yi, A. Iraqi, et al., PCDTBT based solar cells: one year of operation under real-world conditions, Sci. Rep., 2016, 6, 21632-21639.

11 J. H. Bannock, N. D. Treat, M. Chabinyc, N. Stingelin, M. Heeney and J. C. de Mello, The influence of polymer purification on the efficiency of poly(3-hexylthiophene): fullerene organic solar cells, Sci. Rep., 2016, 6, 23651-23658.

12 R. Gangopadhyay and A. De, Conducting polymer nanocomposites: a brief overview, Chem. Mater., 2000, 12(3), 608-622.

13 K. Rajeshwar, N. R. De Tacconi and C. R. Chenthamarakshan, Semiconductor-based composite materials: preparation, properties, and performance, Chem. Mater., 2001, 13(9), 2765-2782.

14 P. Gomez-Romero, Hybrid organic-inorganic materials - in search of synergic activity, Adv. Mater., 2001, 13(3), 163-174.

15 C. Janáky and K. Rajeshwar, The role of (photo) electrochemistry in the rational design of hybrid conducting polymer/semiconductor assemblies: from fundamental concepts to practical applications, Prog. Polym. Sci., 2015, 43, 96-135.

16 X. Ji, Y. Xu, W. Zhang, L. Cui and J. Liu, Review of functionalization, structure and properties of graphene/ polymer composite fibers, Composites, Part A, 2016, 87, 2945.

17 J. Ye, X. Li, J. Zhao, X. Mei and Q. Li, Efficient and stable perovskite solar cells based on functional graphenemodified P3HT hole-transporting layer, RSC Adv., 2016, 6(43), 36356-36361.

18 G. Kaur, R. Adhikari, P. Cass, M. Bown and P. Gunatillake, Electrically conductive polymers and composites for biomedical applications, $R S C A d v ., 2015$, 5(47), 3755337567. 
19 L. K. H. Trang, T. Thanh Tung, T. Young Kim, W. S. Yang, H. Kim and K. S. Suh, Preparation and characterization of graphene composites with conducting polymers, Polym. Int., 2012, 61(1), 93-98.

20 H. S. Dehsari, E. K. Shalamzari, J. N. Gavgani, F. A. Taromi and S. Ghanbary, Efficient preparation of ultralarge graphene oxide using a PEDOT:PSS/GO composite layer as hole transport layer in polymer-based optoelectronic devices, RSC Adv., 2014, 4(98), 55067-55076.

21 M. AngeláRodriguez-Perez, J. de Saja and M. AngeláLopezManchado, Functionalized graphene sheet filled silicone foam nanocomposites, J. Mater. Chem., 2008, 18(19), 22212226.

22 T. Ramanathan, A. Abdala, S. Stankovich, D. Dikin, M. HerreraAlonso, R. Piner, et al., Functionalized graphene sheets for polymer nanocomposites, Nat. Nanotechnol., 2008, 3(6), 327331.

23 S. Stankovich, D. A. Dikin, G. H. Dommett, K. M. Kohlhaas, E. J. Zimney, E. A. Stach, et al., Graphene-based composite materials, Nature, 2006, 442(7100), 282-286.

24 A. Yu, P. Ramesh, M. E. Itkis, E. Bekyarova and R. C. Haddon, Graphite nanoplatelet-epoxy composite thermal interface materials, J. Phys. Chem. C, 2007, 111(21), 7565-7569.

25 P. A. Basnayaka, M. K. Ram, E. K. Stefanakos and A. Kumar, Nanostructured Hybrid Graphene-Conducting Polymers for Electrochemical Supercapacitor Electrodes, in Handbook of Nanoelectrochemistry: Electrochemical Synthesis Methods, Properties, and Characterization Techniques, ed. M. Aliofkhazraei and H. A. S. Makhlouf, Cham: Springer International Publishing, 2016. pp. 479501.

26 A. Chauhan, A. Gusain, P. Jha, S. Koiry, V. Saxena, P. Veerender, et al., Graphene composite for improvement in the conversion efficiency of flexible poly 3-hexylthiophene:[6,6]-phenyl C71 butyric acid methyl ester polymer solar cells, Appl. Phys. Lett., 2014, 104(13), 133901.

27 H. Klauk, Organic thin-film transistors, Chem. Soc. Rev., 2010, 39(7), 2643-2666.

28 P. Jha, S. Koiry, V. Saxena, P. Veerender, A. Gusain, A. Chauhan, et al., Air-stability and bending properties of flexible organic field-effect transistors based on poly[ $\left[N-9^{\prime}\right.$ heptadecanyl-2,7-carbazole-alt-5,5-(4',7'-di-2-thienyl-2' $, 1^{\prime}, 3^{\prime}$ benzothiadiazole)], Org. Electron., 2013, 14(10), 2635-2644.

29 Y. Hao, Y. Wang, L. Wang, Z. Ni, Z. Wang, R. Wang, et al., Probing Layer Number and Stacking Order of Few-Layer Graphene by Raman Spectroscopy, Small, 2010, 6(2), 195200.

30 C. Backes, K. R. Paton, D. Hanlon, S. Yuan, M. I. Katsnelson, J. Houston, et al., Spectroscopic metrics allow in situ measurement of mean size and thickness of liquidexfoliated few-layer graphene nanosheets, Nanoscale, 2016, 8(7), 4311-4323.

31 Y. Y. Wang, Z. H. Ni, T. Yu, Z. X. Shen, H. M. Wang, Y. H. Wu, et al., Raman studies of monolayer graphene: the substrate effect, J. Phys. Chem. C, 2008, 112(29), 10637-10640.

32 M. Iwamoto, T. Manaka, M. Weis and D. Taguchi, Probing and modeling of interfacial carrier motion in organic devices by optical second harmonic generation, J. Vac. Sci. Technol., B: Nanotechnol. Microelectron.: Mater., Process., Meas., Phenom., 2010, 28(4), C5F12-C5F16.

33 T. Manaka, E. Lim, R. Tamura and M. Iwamoto, Direct imaging of carrier motion in organic transistors by optical second-harmonic generation, Nat. Photonics, 2007, 1(10), 581-584.

34 D. Taguchi, M. Weis, T. Manaka and M. Iwamoto, Probing of carrier behavior in organic electroluminescent diode using electric field induced optical second-harmonic generation measurement, Appl. Phys. Lett., 2009, 95(26), 263310.

35 R. Noriega, J. Rivnay, K. Vandewal, F. P. Koch, N. Stingelin, P. Smith, et al., A general relationship between disorder, aggregation and charge transport in conjugated polymers, Nat. Mater., 2013, 12(11), 1038-1044.

36 R. C. Huber, A. S. Ferreira, R. Thompson, D. Kilbride, N. S. Knutson, L. S. Devi, et al., Long-lived photoinduced polaron formation in conjugated polyelectrolyte-fullerene assemblies, Science, 2015, 348(6241), 1340-1343.

$37 \mathrm{~W}$. Chen, H. Huang, A. Thye and S. Wee, Molecular orientation transition of organic thin films on graphite: the effect of intermolecular electrostatic and interfacial dispersion forces, Chem. Commun., 2008, 36, 4276-4278.

38 A. K. Manna and S. K. Pati, Computational Studies on Noncovalent Interactions of Carbon and Boron Fullerenes with Graphene, ChemPhysChem, 2013, 14(9), 1844-1852.

39 J. L. Bredas and G. B. Street, Polarons, bipolarons, and solitons in conducting polymers, Acc. Chem. Res., 1985, 18(10), 309-315.

40 M. J. Deka and D. Chowdhury, Tuning Electrical Properties of Graphene with Different $\pi$-Stacking Organic Molecules, J. Phys. Chem. C, 2016, 120(7), 4121-4129.

41 H. Luo, C. Yu, Z. Liu, G. Zhang, H. Geng, Y. Yi, et al., Remarkable enhancement of charge carrier mobility of conjugated polymer field-effect transistors upon incorporating an ionic additive, Sci. Adv., 2016, 2(5), e1600076.

42 Y. D. Park, J. A. Lim, Y. Jang, M. Hwang, H. S. Lee, D. H. Lee, et al., Enhancement of the field-effect mobility of poly(3hexylthiophene)/functionalized carbon nanotube hybrid transistors, Org. Electron., 2008, 9(3), 317-322.

43 S. H. Park, S. H. Jin, G. H. Jun, S. Jeon and S. H. Hong, Enhanced electrical properties in carbon nanotube/poly(3hexylthiophene) nanocomposites formed through noncovalent functionalization, Nano Res., 2011, 4(11), 11291135.

44 Q. Liu, Z. Liu, X. Zhang, L. Yang, N. Zhang, G. Pan, et al., Polymer Photovoltaic Cells Based on Solution-Processable Graphene and P3HT, Adv. Funct. Mater., 2009, 19(6), 894904.

45 B. Qi and J. Wang, Open-circuit voltage in organic solar cells, J. Mater. Chem., 2012, 22(46), 24315-24325.

46 I.-W. Hwang, D. Moses and A. J. Heeger, Photoinduced Carrier Generation in P3HT/PCBM Bulk Heterojunction Materials, J. Phys. Chem. C, 2008, 112(11), 4350-4354.

47 F. Etzold, I. A. Howard, R. Mauer, M. Meister, T.-D. Kim, K.-S. Lee, et al., Ultrafast Exciton Dissociation Followed by Nongeminate Charge Recombination in PCDTBT:PCBM 
Photovoltaic Blends, J. Am. Chem. Soc., 2011, 133(24), 94699479.

48 D. Veldman, O. Ipek, S. C. Meskers, J. Sweelssen, M. M. Koetse, S. C. Veenstra, et al., Compositional and electric field dependence of the dissociation of charge transfer excitons in alternating polyfluorene copolymer/ fullerene blends, J. Am. Chem. Soc., 2008, 130(24), 77217735.

49 V. Dyakonov and E. Frankevich, On the role played by polaron pairs in photophysical processes in semiconducting polymers, Chem. Phys., 1998, 227(1), 203-217.

50 H. Ohkita, S. Cook, Y. Astuti, W. Duffy, S. Tierney, W. Zhang, et al., Charge carrier formation in polythiophene/fullerene blend films studied by transient absorption spectroscopy, J. Am. Chem. Soc., 2008, 130(10), 3030-3042.

51 A. C. Morteani, P. Sreearunothai, L. M. Herz, R. H. Friend and C. Silva, Exciton regeneration at polymeric semiconductor heterojunctions, Phys. Rev. Lett., 2004, 92(24), 247402.

52 C. Deibel, T. Strobel and V. Dyakonov, Role of the charge transfer state in organic donor-acceptor solar cells, Adv. Mater., 2010, 22(37), 4097-4111.

53 T. M. Burke, S. Sweetnam, K. Vandewal and M. D. McGehee, Beyond Langevin Recombination: How Equilibrium Between Free Carriers and Charge Transfer States Determines the Open-Circuit Voltage of Organic Solar Cells, Adv. Energy Mater., 2015, 5(11), 391-410. 